F. med. Genet. (1966). 3, 159.

\title{
Expectation of Abnormality on Paternal and Maternal Sides: A Computational Model
}

\author{
ELIOT SLATER \\ From the Medical Research Council Psychiatric Genetics Research Unit, Maudsley Hospital, London S.E.5
}

Edwards (1960, 1963) has recently pointed out that conditions which have been attributed to the effects of a single major gene of diminished penetrance are also capable of being explained as the result of polygenic factors, if the condition in question is not very infrequent. It is only necessary to suppose that polygenic factors bring about a predisposition which expresses itself in a recognizable apparently qualitative deviation, once it has passed a given threshold. There is, for instance, evidence that the presence or absence of the hare-lip/cleft palate syndrome is due to polygenic factors (Carter, 1965). It is supposed that there is a critical stage in development by which time the embryonic layers involved must have united; a delay in union short of this point is without effect, but beyond it leaves an incomplete state which is never made good.

Edwards has also pointed out that the two alternative hypotheses are very alike in their effects, so that it is difficult to devise tests to distinguish between them. Nevertheless, it is important to make such a distinction if possible, since they are not equivalent. It has been said that the penetrance of every gene is complete, given the right test of its biochemical action. A hypothesis of major gene action, therefore, suggests investigation along biochemical lines. The polygenic hypothesis is of less heuristic value.

It occurred to the writer that it should be possible to distinguish between polygenic inheritance and the effects of a single dominant major gene with diminished penetrance, by noting the distribution of secondary cases among the relatives on the paternal and maternal sides of the ascendance of affected individuals. On the single gene hypothesis one would expect, in the cases where two or more affected relatives were known, that these would be either on the paternal or the maternal side, but not

Received January 13, 1966. on both. Relatives coming in question would include uncles and aunts, grandparents, and cousins. If polygenic inheritance was operative, the author imagined that a more even distribution between paternal and maternal sides would be found. It was, however, by no means easy to prove this; and when mathematical geneticists, to whom the problem was offered, showed some disinclination to tackle it along rigorous lines, the attempt was made to find an approximate answer by computation carried out on a simplified model.

It is supposed that the many genes predisposing to a condition $A$ are additive in their action; the predisposition to $A$, measured quantitatively, will accordingly be normally distributed. It is also supposed that any individual exceeding $+2 \sigma$ above the population mean is liable to pass the threshold and become recognizable as an $A$-deviant. Whether he actually did so or not, might or might not depend on some additional, possibly accidental, factor.

We now classify fathers and mothers in classes, each class covering a span of $0.5 \sigma$, and its frequency corresponding to the area of the normal curve between the limiting ordinates. Supposing that mating is at random, we are able to calculate the frequency of any given mating as the product of the frequencies of the maternal and paternal classes severally. As a simplifying hypothesis we may then suppose that the children of this mating are distributed normally about the mid-parental value. We take an example from the Table and consider the cell where mothers between the values of $+I \cdot 5$ and +2.00 have mated with fathers between +2.5 and +3.00 . The log maternal frequency is $\overline{2} \cdot 6440159$, the log paternal frequency is $\overline{3} \cdot 6866184$, and the mating frequency is $\overline{4} \cdot 3306343$. The children of this mating are distributed about a mean of $\frac{1}{2}(1 \cdot 75+2 \cdot 75)=2 \cdot 25 \sigma$, and the proportion of them who will deviate from the population mean by $2 \sigma$ or more will correspond to the area of the normal 
TABLE

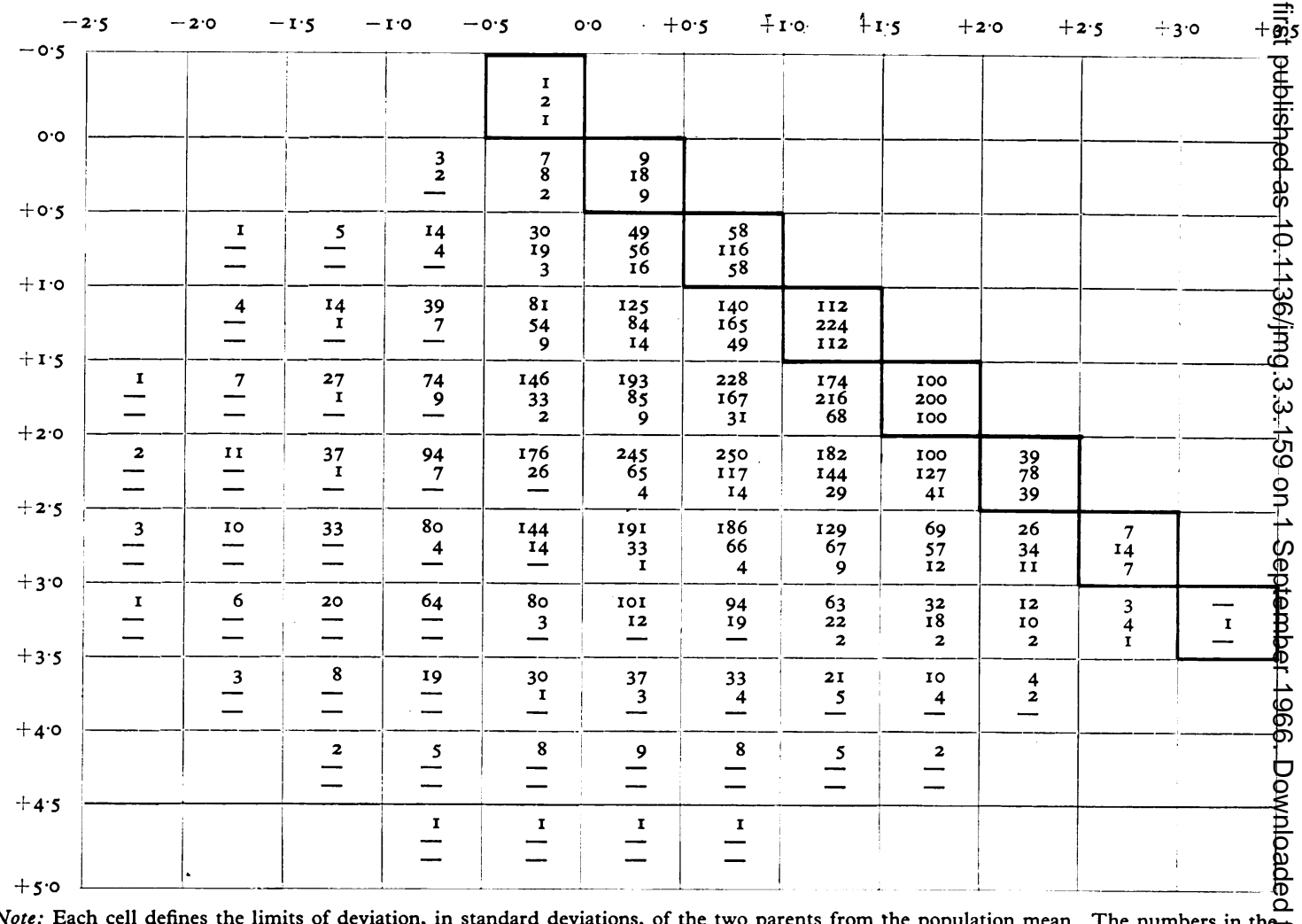

Note: Each cell defines the limits of deviation, in standard deviations, of the two parents from the population mean. The numbers in the cells represent from above downwards the relative probabilities of an individual of such parentage, himself deviating from the population byo $2 \sigma$ or more, having respectively two sibs of a father, one sib of a father and one sib of a mother, and two sibs of a mother, both deviating from the population mean by $2 \sigma$ or more.

curve to the positive side of $-0.25 \sigma$, the log frequency being $\overline{\mathrm{I}} \cdot 7772139$. The contribution of this mating to our observed propositi may, therefore, be estimated, in comparison with the contributions of all other cells, as $\overline{4} \cdot 1078482$.

We now have to make some rough-and-ready assumptions about the paternal and maternal relatives. Uncles and aunts will tend to be distributed about means of +0.875 on the maternal and +1.375 on the paternal side; mothers and fathers, of course, deviate by twice those margins, but more distant relatives by a lesser amount. At this point we may treat all relatives as if they were sibs of the parents. Maternal relatives will accordingly need to exceed their expected mean by $1 \cdot 125 \sigma$, and paternal relatives by $0.625 \sigma$, to attain the critical value of $+2.0 \sigma$. We may call the probabilities of these deviations respectively $m$ and $p$, with $\log$ values in this case of $\overline{\mathrm{I}} \cdot \mathrm{I} 149360$ and $\overline{\mathrm{I}} \cdot 4248623$, respectively.

We now consider the relative probabilities of finding two affected relatives on the paternal side of finding one paternal and one maternal affecte $\bar{B}$ relative, and of finding two affected maternats relatives. Supposing that $n$ relatives are examined on each side, these probabilities reduce to being propor? tionally $(n-\mathrm{I}) p^{2}(\mathrm{I}-m)^{2}, 2 n p m(\mathrm{I}-\mathrm{p})(\mathrm{I}-\mathrm{m})$ and $(n-1) m^{2}(1-p)^{2}$. Omitting coefficients it terms of $n$, they are in the cell we have instanced in $\log$ form $\overline{2} \cdot 7284660, \overline{2} \cdot 6459025$, and $\overline{3} \cdot 96127909$ respectively. Adding to these the log of the ceff: probability, $\overline{4} \cdot 1078482$, we get figures whose antilog are respectively $6.9,5.7$, and $\mathrm{I} \cdot 2 \times 10^{-6}$. The Tables has been so prepared as to include all matings which gave a value for the log cell probability added to the $\log$ of $p^{2}(\mathrm{I}-m)^{2}$ in excess of $\overline{7} \cdot 0$, stretching over three orders of magnitude. The probabilities of on paternal and one maternal relative affected, and fo two maternal relatives affected, are entered also where they exceed this figure.

We may now use this table to compute th relative expectations of finding two paternal relative 
affected, two maternal relatives, or one of each. The section of the bivariate expanse shown in the Table should have been extended symmetrically on the other side of the diagonal marked in heavy outlines, so that paternal figures become maternal ones and vice versa. Adding up the figures for the entire area so described we obtain the numbers $4675: 4193: 4675$, or approximately I I : IO : I I.

A similar computation was made on the basis that all relatives were equivalent to mothers or fathers. This produced the proportions 3 I $233: 12454: 31233$, or approximately $5: 2: 5$. It was not thought necessary to make yet another computation, to take care of the cases in which information was obtained about more remote relatives. In their case, the proportion of cases in which both a paternal and a maternal relative were ascertained should exceed the proportion of cases in which two paternal, alternatively maternal, relatives were ascertained.

We now have to take account of the value we set on $n$. In the limiting case, in which $n$ is large, $n$ and $(n-\mathrm{I})$ approach equality, and the proportions above noted remain undisturbed. At the other limit, where $n=2$, the expected proportion of cases in which one paternal and one maternal relative are ascertained has to be doubled relatively to unilateral distributions. In practical application, one could make an estimate of the mean number of paternal and maternal relatives per family, on which information was available, in the course of collecting the information designed to test the alternative hypotheses of polygenic or monogenic inheritance. This empirically found value of $n$ could then be used to adjust the expectations against which comparison was to be made.

\section{Summary}

It is difficult to distinguish the effects of inheritance by a dominant major gene with diminished penetrance from polygenic inheritance. Nevertheless, it seems to be worth while making the distinction in the case of genetically determined abnormalities, if this is practicable. The possibility has been considered that, if families are sought for in which relatives on the paternal or maternal side include two or more secondary cases of the condition under investigation, single gene inheritance might be exhibited in a preponderance of cases on either the paternal or maternal side, while polygenic inheritance might tend to go with a more even distribution of secondary cases between the two sides of the ascendance. A computational model, making use of some simplifying hypotheses, has been set up to test this possibility. It is found that, in the cases of near relatives such as parents or sibs of parents, even with polygenic inheritance, one expects a unilateral preponderance of secondary cases, rather than a more even distribution. This does not exclude the possibility of distinguishing between the consequences of the two alternative hypotheses, if reliable accounts of more distant relatives are available, or if use can be made of families with three or more secondary cases.

\section{REFERENCES}

Carter, C. O. (1965). The inheritance of common congenital malformations. Progr. med. Genet., 4, 59.

Edwards, J. H. (1960). The simulation of Mendelism. Acta genet. (Basel), 10, 63.

(1963). The genetic basis of common disease. Amer. F. Med., 34,627 . 